\title{
Avaliação da longitudinalidade em unidades básicas de saúde tradicionais e com estratégia de saúde da família utilizando o Primary Care Assessment Tool (PCATool)
}

\author{
Evaluation of longitudinality in traditional basic health units or with family health strategy \\ using the Primary Care Assessment Tool (PCATool)
}

Evaluación de la longitudinalidad en las unidades básicas de salud tradicionales o con la estrategia de salud familiar utilizando Primary Care Assessment Tool (PCATool)

André Vinícius de Oliveira ${ }^{1 *}$, Barbara Correia Neves Sabino¹, Luis Regagnan Dias ${ }^{1}$, Ana Beatriz Viana Gouvea1, Giulia Ugucioni Garbelini', Isabela Galliazzi Paiva¹, Larissa de Assis Timpone ${ }^{1}$, Paulo Ricardo Alves Moreira1, Leonardo Vieira de Lima1', Paulo Appollonio Filho'1.

\section{RESUMO}

Objetivo: Avaliar e comparar a qualidade da longitudinalidade ofertada em unidades básicas de saúde (UBS) com e sem estratégia de saúde da família (ESF). Métodos: Estudo transversal, de abordagem quantitativa realizado na cidade de Rio verde. Utilizou-se o questionário Primary Care Assessment Tool (PCATool)-Brasil versão adulto e uma questionário sociodemográfico. A coleta de dados foi realizada nas UBS estudadas através de usuários do SUS de 18 a 65 anos. Após a coleta utilizou-se o programa SPSS para aplicar o teste t de student para análise das médias entre as variáveis utilizadas. Resultados: As UBS com ESF obtiveram escore médio de 6,99, e as tradicionais, 6,47, mas sem significância estatística entre esses dois grupos. A média entre todas as UBS foi de 6,77 o que satisfaz o ponto de corte estabelecido na literatura que considera satisfatória uma média $\geq 6,6$. Verificou-se ainda que 5 dos 14 quesitos da longitudinalidade abordados no PCATool foram maiores entre as UBS com ESF com $p<0,05$. Conclusão: As UBS com ESF apresentaram desempenho moderadamente superior às tradicionais e avaliação geral condiz com o encontrado em outros estudos similares.

Palavras-Chave: Atenção primária à saúde, Garantia da qualidade dos cuidados de saúde, Continuidade da assistência ao paciente.

\begin{abstract}
Objective: To evaluate and compare the quality of longitudinality offered in basic health units (UBS) with and without a family health strategy (FHS). Methods: Cross-sectional study with a quantitative approach carried out in the city of Rio verde. The adult version of the Primary Care Assessment Tool (PCATool)-Brazil questionnaire and a sociodemographic questionnaire were used. Data collection was performed in the UBS studied through SUS users aged 18 to 65 years. After the collection, the SPSS program was used to apply the Student's t test to analyze the means between the variables used. Results: The UBS with FHS obtained an average score of 6.99 , and the traditional ones, 6.47 , but without statistical significance between these two groups. The average among all UBS was 6.77 , which satisfies the cutoff point established in the literature, which considers a mean $\geq 6.6$ satisfactory. It was also found that 5 of the 14 longitudinality issues addressed in the PCATool were higher among UBS with ESF with $p<0.05$. Conclusion: The UBS with FHS presented a performance moderately superior to the traditional ones and the general assessment is consistent with that found in other similar studies.
\end{abstract}

Key words: Primary health care, Quality assurance, Continuity of patient care.

1 Universidade de Rio Verde (UNIRV), Rio Verde - GO. *E-mail: av384993@gmail.com 


\section{RESUMEN}

Objetivo: Evaluar y comparar la calidad de la longitudinalidad ofrecida en las unidades básicas de salud (UBS) con y sin una estrategia de salud familiar (FHS). Métodos: Estudio transversal con un enfoque cuantitativo realizado en la ciudad de Río Verde. Se utilizó la versión para adultos del cuestionario Primary Care Assessment Tool (PCATool)-Brasil y un cuestionario sociodemográfico. La recolección de datos se realizó en la UBS estudiada a través de usuarios del SUS de 18 a 65 años. Después de la recolección, se utilizó el programa SPSS para aplicar la prueba t de Student para analizar las medias entre las variables utilizadas. Resultados: La UBS con FHS obtuvo un puntaje promedio de 6.99, y los tradicionales, 6.47, pero sin significación estadística entre estos dos grupos. El promedio entre todos los UBS fue de 6.77, que satisface el punto de corte establecido en la literatura, que considera una media $\geq 6.6$ satisfactoria. También se encontró que 5 de los 14 problemas de longitudinalidad abordados en PCATool fueron mayores entre UBS con FSE con $p<0.05$. Conclusión: La UBS con FHS presentó un desempeño moderadamente superior a los tradicionales y la evaluación general es consistente con la encontrada en otros estudios similares.

Palabras clave: Atención primaria de salud, Garantía de la calidad de atención de salud, Continuidad de la atención al paciente.

\section{INTRODUÇÃO}

Em 1978, com a Conferência Internacional de Alma-Ata, a Atenção primária à saúde (APS), ganhou força como estratégia a ser utilizada nos serviços de saúde em todo mundo, através de uma assistência contínua, contemplando, prevenção, promoção, cura e reabilitação a custos possíveis.

Já no Brasil, a partir de 1988 com a implantação do sistema único de saúde (SUS) houve uma grande ampliação da população atendida e do número de estabelecimentos expandindo a oferta de serviços. Estes foram os primeiros passos para a melhoria da atenção primária à saúde, em 1994 foi criado o programa de saúde da família (PSF) a partir de experiências em algumas cidades do Brasil, e em 1998 este programa passa a ser considerado uma estratégia estruturante do sistema de saúde (MINISTÉRIO DA SAÚDE, 2002).

Em 2006 com a aprovação da Política Nacional de Atenção Básica (PNAB), pela portaria ํㅡ 648 foram determinadas as diretrizes e normas para a organização da Atenção Básica, que em seguida foram atualizadas pela portaria nำ2488/2011 e ㄲo2436/2017 (DIARIO OFICIAL, 2006).

A atenção primária à saúde, definido pelo PNAB como sinônimo de atenção básica $(A B)$, e a porta de entrada preferencial no SUS, sendo definida como as ações de saúde, individuais e coletivas, abrangendo a promoção e proteção da saúde, prevenção agravos, diagnóstico, tratamento, reabilitação, redução de danos e manutenção da saúde. É baseada na atenção integral, impactando a situação de saúde das coletividades, a partir dela o SUS pode otimizar os recursos e reduzir os gastos com serviços especializados (TURCI MA, et al., 2015; BRASÍLIA, 2010).

Nesse contexto os gestores municipais continuamente aderem a Estratégia de Saúde da Família (ESF), que é composta por uma equipe multidisciplinar que atua principalmente na prevenção e promoção de saúde, como forma de estruturar a APS. Porém, a adesão à ESF em grande parte do país não garante a mudança no modelo hospitalocêntrico e curativista, segundo alguns autores isso se deve à falta de preparação dos profissionais de saúde e a falta de recursos (ALMEIDA MCP e MISHIMA SM, 2001).

Além disso o desenvolvimento da APS se dá de forma heterogênea no país, com diferentes níveis de qualidade e problemas a serem enfrentados pelas equipes, o que torna mais difícil a correta adequação de recursos por parte dos gestores (PRATES ML, et al., 2017).

A APS foi dividida por Starfield B (2004) em 4 atributos essenciais: acessibilidade, longitudinalidade, integralidade e coordenação da atenção e 2 atributos derivados: atenção centrada na família e orientação comunitária. Essa concepção também é notada no texto da PNAB, indicando uma convergência entre os documentos do ministério da saúde (MS) e a proposta de Starfield B (2004). 
O atributo longitudinalidade, que é o destaque deste estudo, pode ser entendido como o acompanhamento dos usuários pelos profissionais de saúde de forma contínua ao longo do tempo, refletindo em uma relação de confiança entre profissionais e usuários sendo que algumas vezes longitudinalidade é substituída pelo termo "continuidade do cuidado" (STARFIELD B., 2004). Esta continuidade do cuidado permite um maior conhecimento das equipes sobre os pacientes adstritos possibilitando diagnósticos mais precisos, maior adesão ao tratamento e aumentando a resolutividade do sistema de saúde (ALMEIDA MCP e MISHIMA SM, 2001). Além disso o fortalecimento do vínculo entre o profissional e o paciente permite que as condutas sejam baseadas no indivíduo como um todo e não apenas na sua patologia (STARFIELD B., 2004).

O instrumento utilizado para avaliar a longitudinalidade foi o Instrumento de Avaliação da Atenção Primária Primary Care Assessment Tool (PCATool), o qual foi adaptado à realidade brasileira, tornando-se o PCAToolBrasil, levando em conta características culturais da população e do sistema nacional de saúde, a versão utilizada foi a do usuário adulto. (BRASÍLIA., 2010). Esse instrumento já foi utilizado e validado em vários países em estudos com diferentes características e objetivos e utilizado em diversas regiões do país o que permite estabelecer comparações dos resultados obtidos nesta pesquisa com o encontrado em outras regiões e condições de estudo (PRATES ML, et al., 2017).

Além disso o PCATool é um instrumento que permite uma avaliação mais próxima da saúde da família e contém um manual do MS para a utilização do instrumento adaptado à realidade brasileira. Nesse sentido o MS recomenda que esse instrumento seja utilizado na avaliação e monitoramento da APS como uma rotina das Equipes de saúde da família, por diversos níveis de gestão e para uso acadêmico (PRATES ML, et al., 2017; HAUSER L, et al., 2013; BRASÍLIA, 2010).

Com isso, este estudo ao avaliar a qualidade da longitudinalidade na APS teve por objetivo contribuir para o conhecimento da atenção básica no Brasil. Além disso buscou entender qual a influência da ESF nas UBS e como outros aspectos sociodemográficos dos participantes se relacionam com o nível de longitudinalidade obtido.

\section{MÉTODOS}

Este é um estudo transversal de base populacional e abordagem quantitativa que foi realizado no município de Rio Verde Goiás, localizado no interior do estado de Goiás com população estimada de 235 mil pessoas e possui um total de 14 unidades básicas de saúde. A coleta de dados ocorreu no último trimestre de 2019, foram entrevistados 8 usuários por UBS, totalizando 112 participantes.

Os participantes foram selecionados ao acaso na fila de atendimento da UBS. Os critérios de inclusão foram: ter entre 18 e 65 anos, ser usuário da UBS na qual estava ocorrendo a entrevista, compreender e assinar o termo de consentimento livre e esclarecido (TCLE) e os critérios de exclusão: não aceitar participar da pesquisa, não responder o questionário completamente, não ter sido atendido previamente na UBS da entrevista. O questionário sociodemográfico aplicado versa sobre aspectos psicossociais como idade, sexo, escolaridade, renda familiar e intensidade do vínculo com a UBS que compreende a frequência de comparecimento a UBS e participação de grupos de educação em saúde. Por fim, do Instrumento de Avaliação da Atenção Primária (PCATool - Primary Care Assessment Tool), utilizou-se as 14 questões do atributo longitudinalidade (Tabela 1).

No PCATool as respostas de cada item variam em uma escala tipo likert: 1 (com certeza não), 2 (provavelmente não), 3 (provavelmente sim), 4 (com certeza sim) e 9 (não sei não lembro). Caso as respostas "não sei/não lembro" atingirem mais de $50 \%$ das respostas os dados deste indivíduo eram deixados em branco não sendo considerados no cálculo final, se menos de $50 \%$ das respostas foram "não sei/não lembro" estas eram substituídas por "provavelmente não" e consideradas no cálculo final conforme proposto pelo manual do MS para o PCATool-Brasil (BRASÍLIA, 2010).

A escala de 1 a 4 foi transformada em uma de 0 a 10, a partir disso os resultados foram dicotomizados em scores. Sendo alto quando $\geq 6,6$ o que corresponde as duas respostas superiores e score baixo quando $\leq 6,6$ que corresponde as duas respostas inferiores possibilitando a comparação dos resultados da pesquisa com os dados disponíveis na literatura (PRATES ML, et al., 2017). O item 14 "Você mudaria sua UBS/ou 
médico/enfermeiro para outro serviço de saúde se isto fosse muito fácil de fazer?" diferente de todos os outros itens possui maior valor quanto pior a avaliação da longitudinalidade, pois quanto maior o valor atribuído, mais facilmente o participante trocaria sua UBS por outra, por não ter um bom vínculo com a UBS que frequenta, assim seus valores foram invertidos antes de serem inseridos no Excel, para que dessa forma, 0 seu valor seja diretamente proporcional ao nível de longitudinalidade, após isso foi feito a média de todos os 14 itens e obteve-se o escore médio da longitudinalidade.

Os dados foram digitados em planilha do Excel, manipulados e posteriormente inseridos no programa estatístico Statistical Package for Social Science (SPSS) versão 22.0.0.0. Além disso, para se estabelecer correlações entre o questionário sociodemográfico e os quesitos do PCATool, e entre as médias do PCATool entre as diferentes UBS, com e sem ESF, foi utilizado o teste t de student.

Esta pesquisa seguiu em todos os seus estágios os princípios éticos para pesquisas envolvendo seres humanos descritos pela resolução do conselho nacional de saúde № 466, de 12 de dezembro de 2012 (BRASIL., 2013). O projeto foi aprovado pelo Comitê de Ética em Pesquisa - CEP da UNIRV (protocolo: 08560819.3.0000.5077).

\section{RESULTADOS E DISCUSSÃO}

Referente aos dados sóciodemoráficos, a idade média dos sujeitos foi de 43,81 anos e uma maior idade foi associada a uma melhor avaliação dos itens: D9 "O seu médico/enfermeiro sabe quais problemas são mais importantes para você?" e D11 "O seu médico/enfermeiro sabe a respeito do seu trabalho ou emprego?" e pior avaliação do item D8 "O seu médico/enfermeiro sabe quem mora com você?", ambos com significância $p<0,05$.

Quanto ao sexo houve predominância de mulheres, assim como na maioria dos estudos de avaliação da APS, sendo neste, 85 mulheres e 27 homens, não havendo diferenças estatisticamente significativas em relação ao gênero (GOMES MFP e FRACOLLI LA, 2018) .

Além disso, 44,6\% tinham como escolaridade o ensino fundamental completo e $32 \%$ o ensino médio, observou-se, que uma escolaridade mais elevada se correlacionou com maior escore no item D5 "O seu médico/enfermeiro Ihe dá tempo suficiente para falar sobre as suas preocupações ou problemas?" com $p=0,002$, o que poderia ser reflexo do melhor entendimento do usuário da importância do profissional esgotar todas as queixas do paciente, essa relação também foi verdadeira em outro estudo em que a maior escolaridade se associou a maior escore da ESF (LEÃO CDA e CALDEIRA AP, 2011). No entanto maior escolaridade também se associou a menor nota no item D1 "Quando você vai a sua UBS e sempre o mesmo médico ou enfermeiro que atende você todas às vezes", com $p=0,046$.

A análise da renda familiar por pessoa revelou que $49,1 \%$ recebiam até 1 salário mínimo e $48,25 \%$ entre 1 e 2 salários mínimos. Quanto a frequência de consultas desses indivíduos nas UBS, $47 \%$ vão de 3 a 5 vezes ao ano e $45 \%$ vão 2 vezes ou menos em um ano.

Uma maior frequência se correlacionou com maior pontuação no item D3 "O seu médico/enfermeiro responde suas perguntas de maneira que você entenda?" $p=0,001$, e D12 "O seu médico/enfermeiro saberia de alguma forma se você tivesse problemas em obter ou pagar por medicamentos que você precisa?" com significância de $p=0,025$, o que pode evidenciar uma melhor comunicação entre as partes, pelo vínculo já estabelecido. Porém, o quesito D6 "Você se sente à vontade contando as suas preocupações ou problemas ao seu médico/enfermeiro?" teve pior resultado com um maior número de consultas $(p=0,005)$.

Além disso apenas 2 participantes (1,7\%), frequentavam grupos de educação em saúde. $O$ grupo de educação em saúde e apontado como um espaço relevante para explicações dos profissionais de saúde aos usuários e importante também para a troca de conhecimentos e vivencias entre os pacientes (SILVA FM, et al., 2014).

No quesito D1 "Quando você vai a sua UBS e sempre o mesmo médico ou enfermeiro que atende você todas às vezes" o escore médio foi de 6,66, no limite do inferior do score alto, sendo de 7,0 em UBS com ESF e de 6,1 nas UBS tradicionais, $p=0,006$ (Tabela 1, Tabela 2). 
Tabela 1 - Quesitos do atributo longitudinalidade.

\begin{tabular}{cl}
\hline D1 & $\begin{array}{l}\text { Quando você vai a sua UBS é sempre o mesmo médico ou enfermeiro que atende você todas as } \\
\text { vezes? }\end{array}$ \\
\hline D2 & Você acha que o seu médico/enfermeiro entende o que você diz ou pergunta? \\
\hline D3 & O seu médico/enfermeiro responde suas perguntas de maneira que você entenda? \\
\hline D4 & $\begin{array}{l}\text { Se você tiver uma pergunta, pode telefonar e falar com o médico ou enfermeiro que melhor } \\
\text { conhece você? }\end{array}$ \\
\hline D5 & $\begin{array}{l}\text { O seu médico/enfermeiro lhe dá tempo suficiente para falar sobre as suas preocupações ou } \\
\text { problemas? }\end{array}$ \\
\hline D6 & $\begin{array}{l}\text { Você se sente à vontade contando as suas preocupações ou problemas ao seu } \\
\text { médico/enfermeiro? }\end{array}$ \\
\hline D7 & $\begin{array}{l}\text { O seu médico/enfermeiro conhece você mais como pessoa do que somente como alguém com um } \\
\text { problema de saúde? }\end{array}$ \\
\hline D8 & O seu médico/enfermeiro sabe quem mora com você? \\
\hline D9 & O seu médico/enfermeiro sabe quais problemas são mais importantes para você? \\
\hline D10 & O seu médico/enfermeiro conhece a sua história clínica (história médica) completa? \\
\hline D11 & O seu médico/enfermeiro sabe a respeito do seu trabalho ou emprego? \\
\hline D12 & $\begin{array}{l}\text { O seu médico/enfermeiro saberia de alguma forma se você tivesse problemas em obter ou pagar } \\
\text { por medicamentos que você precisa? }\end{array}$ \\
\hline D13 & O seu médico/enfermeiro sabe a respeito de todos os medicamentos que você está tomando? \\
\hline D14 & $\begin{array}{l}\text { Você mudaria sua UBS / ou médico/ enfermeiro" para outro serviço de saúde se isto fosse muito } \\
\text { fácil de fazer? }\end{array}$ \\
\hline Fonte: Oliveira AV et al, 2020. Baseado em: Manual do Instrumento de Avaliação da Atenção Primária à
\end{tabular}

Fonte: Oliveira AV, et al., 2020. Baseado em: Manual do Instrumento de Avaliação da Atenção Primária à Saúde (BRASÍLIA, 2010).

Tabela 2 - Escore de 0 a 10 dos quesitos do atributo longitudinalidade atraves do PCATool em UBS com ESF e UBS tradicionais. Rio Verde, GO, 2020.

\begin{tabular}{cccccc}
\hline Variáveis & UBS com ESF & UBS sem ESF & Teste “ $t$ " & p-valor & UBS com e sem ESF \\
\hline \multicolumn{7}{c}{ (Média e DP) } & (Média e DP) & & & Média \\
\hline D2 & $7,0 \pm 2,8$ & $6,1 \pm 3,7$ & 1,46 & 0,006 & 6,66 \\
\hline D3 & $8,8 \pm 1,8$ & $8,6 \pm 2,1$ & 0,64 & 0,255 & 8,75 \\
\hline D4 & $8,6 \pm 2,1$ & $9,0 \pm 1,9$ & $-0,84$ & 0,185 & 8,83 \\
\hline D5 & $8,9 \pm 4$ & $5,5 \pm 3,9$ & $-2,10$ & 0,581 & 4,63 \\
\hline D6 & $9,1 \pm 2,5$ & $7,5 \pm 3,4$ & 2,32 & 0,005 & 8,3 \\
\hline D7 & $6,7 \pm 3,5$ & $8,6 \pm 2,1$ & 1,22 & 0.030 & 8,9 \\
\hline D8 & $3,4 \pm 4,2$ & $4,4 \pm 3,3$ & 0,38 & 0,418 & 6,6 \\
\hline D9 & $9,1 \pm 1,7$ & $8,5 \pm 2,3$ & 1,48 & 0,009 & 8,8 \\
\hline D10 & $7,0 \pm 3,2$ & $6,9 \pm 3,4$ & 0,22 & 0,753 & 7 \\
\hline D11 & $3,2 \pm 3,9$ & $1,6 \pm 2,6$ & 2,46 & 0,000 & 2,55 \\
\hline D12 & $4,7 \pm 3,5$ & $2,7 \pm 3,4$ & 3,01 & 0,426 & 3,86 \\
\hline D13 & $8,1 \pm 2,5$ & $6,5 \pm 3,4$ & 2,76 & 0,085 & 7,5 \\
\hline D14 & $8,6 \pm 2,5$ & $7,9 \pm 2,8$ & 1,42 & 0,102 & 8,33
\end{tabular}

Fonte: Oliveira AV, et al., 2020.

O acompanhamento de forma regular pela mesma equipe, isoladamente, já facilita a criação de vínculos e confiança, promovendo diagnósticos mais precisos, tratamentos mais eficazes e maior resolutividade. (STARFIELD B, 2004) A estabilidade dos profissionais nas UBS é considerado uma fator fundamental para a qualidade da APS, por ser crucial na continuidade do cuidado entre o profissional e o usuário, sem essa condição, os outros quesitos avaliados ficam prejudicados pela fragilidade da relação entre o profissional e usuário (CUNHA EM e GIOVANELLA L, 2011). 
O quesito D2 "Você acha que o seu médico/enfermeiro entende o que você diz ou pergunta?", D3 "O seu médico/enfermeiro responde suas perguntas de maneira que você entenda?" e D6 "Você se sente à vontade contando as suas preocupações ou problemas ao seu médico/enfermeiro?", avaliam em última análise a qualidade da comunicação durante as consultas. Os quesitos D2 e D6 tiveram maior média nas UBS com ESF e D3 nas tradicionais, porém apenas o item D6 teve significância estatística entre médias, todos esses itens tiveram uma boa avaliação geral, conforme mostra a Tabela 2.

A relação de confiança e o vínculo estabelecido na longitudinalidade da APS é constituida dentre outras formas pelo diálogo, assim desenvolver a escuta, utilizar linguagem acessível, identificar de forma precisa as necessidades e valorizar as queixas são ferramentas essenciais para uma continuidade do cuidado adequada. (RIBEIRO LCC, et al., 2010). Nesse sentido o profissional terá maior chance de compreender o paciente como indivíduo, estimulando sua participação na tomada de decisões, respeitando assim a autonomia do indivíduo, o que aumenta as chances do paciente de aderir ao tratamento (STARFIELD B, 2004).

No item D4 "Se você tiver uma pergunta, pode telefonar e falar com o médico ou enfermeiro que melhor conhece você?" obteve-se um score médio de 2,39 , o que pode traduzir uma fragilidade no vínculo entre médico e paciente. A comunicação por telefone ou outros meios de comunicação permite esclarecer dúvidas sobre o tratamento, estimula o vínculo entre profissional e usuário e pode resultar em um menor número de consultas, com as dúvidas sendo esclarecidas por telefone (STARFIELD B, 2004).

Em D5 "O seu médico/enfermeiro lhe dá tempo suficiente para falar sobre as suas preocupações ou problemas?" a média geral foi de 8,3 sendo maior na UBS com ESF, 8,9 contra 7,6 nas tradicionais, de forma estatisticamente significante, $p=0,005$. Esse quesito interfere de forma direta na capacidade de uma comunicação adequada entre profissional e usuário, além disso um maior tempo de atendimento estimula um acolhimento de forma holística do paciente a medida que permite esgotar suas queixas, dúvidas e facilita a compreensão de aspectos pessoais não se limitando apenas ao processo saúde doença (BARATIERI T e MARCON SS, 2012; STARFIELD B, 2004).

Os itens D7 "O seu médico/enfermeiro conhece você mais como pessoa do que somente como alguém com um problema de saúde?" e D9 "O seu médico/enfermeiro sabe quais problemas são mais importantes para você?", tiveram avaliação positiva, $\geq 6,6$, sendo maiores na UBS com ESF, com significância estatística no item $D 9, p=0,009$. Estes itens refletem, através da percepção do paciente a capacidade do profissional de entender o indivíduo além da sua patologia, propondo tratamentos para o sujeito inserido em seu contexto e levando em consideração o que mais o aflige.

No que concerne aos itens D8 "O seu médico/enfermeiro sabe quem mora com você?", D11 "O seu médico/enfermeiro sabe a respeito do seu trabalho ou emprego?" e D12 "O seu médico/enfermeiro saberia de alguma forma se você tivesse problemas em obter ou pagar por medicamentos que você precisa?" obtevese resultados negativos, sendo que em D11 e D12 a média foi maior nas UBS com ESF, sendo estatisticamente significante em D11. Essas informações contribuem para o entendimento por parte do profissional do contexto familiar e social do usuário, aumentando as chances de obter sucesso no diagnóstico e tratamento, garantindo, por exemplo, que o usuário vai ter possibilidade de obter os medicamentos prescritos e caso não tenha adequar novas condutas. Nesse sentido estes resultados negativos vão em desacordo com as propostas da APS. (LINARD AG, 2011)

Em D10 "O seu médico/enfermeiro conhece a sua história clínica (história médica) completa?" e D13 "O seu médico/enfermeiro sabe a respeito de todos os medicamentos que você está tomando?" houve uma avaliação de respectivamente: 7 e 7,5, ambos maiores entres as UBS com ESF. A importância desses aspectos se traduzem pelas possíveis implicações no processo de diagnóstico, tratamento e interações medicamentosas revelados através dessas informações.

Por fim o quesito D14 "Você mudaria sua UBS / ou médico/ enfermeiro" para outro serviço de saúde se isto fosse muito fácil de fazer?" que teve seus valores invertidos: 4(com certeza sim) vira 1, 3(provavelmente sim) vira 2, 2 (provavelmente não) vira 3 e 1(com certeza não) vira 4, para que o score tivese um valor maior quanto maior for a longitudinalidade, é após transformar essa ecala de 1 a 4 em uma de 0 a 10, obteve- 
se uma média de 8,33, esse quesito reflete diretamente o grau de filiação do usuário, que se obtiver uma boa relação com os profissionais do serviço, tenderão a continuar neste mesmo estabelecimento (BARATIERI T e MARCON SS, 2012).

A média entre os 14 quesitos foi de 6,77, sendo 6,99 e 6,47 nas UBS com e sem ESF respectivamente, assim de forma geral as UBS com ESF foram superiores, porém isso foi estatisticamente significativo em apenas 5 quesitos. Quanto a avaliação geral da longitudinalidade da APS, apesar de estar no limite superior da avaliação, z6,6, 4 dos 14 quesitos estão abaixo dessa meta.

Dessa forma a despeito da avaliação geral ter sido $\geq 6,6$, o fato da média estar nesse limite estabelecido e 4 itens abaixo dele, demonstra que as práticas desenvolvidas na APS nessa região, ainda são em boa parte baseado no modelo biomédico curativo. Este fato pode se dever a forma como os profissionais direcionam a assistência para a doença e não para a pessoa, favorecem o número de atendimentos em detrimento da qualidade, dificultando a formação de vínculos (STARFIELD B, 2004)

Ao compararmos as médias entre as 14 UBS avaliadas, observa-se diferenças significativas como demonstrado na Tabela 3, onde a melhor avaliação foi de 8,3 e a pior de 4,6. O que está de acordo com os dados encontrados em outros estudos que demonstraram grande variabilidade no nível da longitudinalidade da APS ofertado em um mesmo município o que pode ser explicado por fatores estruturais: disponibilidade de equipamentos, tempo dos profissionais na equipe, número de equipes por UBS, tempo de permanência do médico na unidade (TURCI LM, et al., 2015; OLIVEIRA MPR, et al., 2016) (Tabela 3).

Tabela 3 - Escore geral de 0 a 10 do atributo do PCATool em UBS. Rio Verde, GO, 2020.

\begin{tabular}{cc}
\hline Variáveis & Média e DP \\
\hline UBS 1 & $6,78 \pm 0,74$ \\
UBS 2 & $6,57 \pm 1,00$ \\
UBS 3 & $6,84 \pm 0,96$ \\
UBS 4 & $8,00 \pm 0,74$ \\
UBS 5 & $5,71 \pm 0,91$ \\
UBS 6 & $8,24 \pm 0,46$ \\
UBS 7 & $8,36 \pm 0,26$ \\
UBS 8 & $5,38 \pm 0,90$ \\
UBS 9 & $7,08 \pm 0,84$ \\
UBS 10 & $5,98 \pm 1,01$ \\
UBS 11 & $7,70 \pm 0,67$ \\
UBS 12 & $4,67 \pm 0,97$ \\
UBS 13 & $6,99 \pm 0,78$ \\
UBS 14 & $6,42 \pm 0,64$ \\
\hline
\end{tabular}

Fonte: Oliveira AV, et al.,2020.

A necessidade de uma formação específica na APS, para um AB adequada pode refletir uma insuficiência dos cursos de graduação de realizar mudanças no ensino de acordo com as necessidades atuais da atenção básica (LEÃO CDA, CALDEIRA AP, 2011).

Ao analisarmos às médias de longitudinalidade avaliadas pelo PCATool encontrados na literatura, não a uniformidade nas médias obtidas nos diferentes estudos em todo o Brasil, no entanto na maioria dos estudos, os resultados, são iguais ou superiores a meta estabelecida $(6,6)$, o que está de acordo com o obtido nesse estudo (PRATES ML, et al., 2017).

Este estudo avaliou apenas um dos atributos propostos por Starfield B (2004), nesse sentido outros atributos podem ser avaliados a fim de melhorar o entendimento da APS na região estudada, identificando aspectos frágeis, e que podem direcionar políticas para a melhoria da APS. O entendimento dos fatores que se relacionam com uma boa avaliação da APS, como aspectos estruturais e formação dos profissionais como avaliado por Turci LC (2015) e Oliveira MPR (2016), contribui para o gerenciamento dos recursos de forma a legitimar a APS oferecida como porta de entrada do sistema de saúde. 
Cabe ressaltar que o instrumento a ser utilizado avalia a percepção do usuário que como demonstrado por Elias PE (2006) tende a ter uma avaliação inferior à dos profissionais. Nesse sentido a avaliação sobre a ótica dos profissionais contribui para uma avaliação mais global da APS, podendo ser objetivo de estudos futuros.

\section{CONCLUSÃO}

A partir dos resultados obtidos neste estudo, inferimos que as UBS com ESF tiveram avaliação superior as UBS tradicionais, com score de 6,99 e 6,47 nas UBS com e sem ESF respectivamente, sendo que 0 score superior nas UBS com ESF foi estatisticamente significativa em D1, D5, D6, D9 e D11. No entanto avaliação geral da APS demonstra a necessidade da melhora do atendimento em alguns quesitos do atributo longitudinalidade a fim de aplicar de forma integral as diretrizes propostas pela PNAB para as ESFs. Por fim a conclusão deste estudo demonstra informações específicas da longitudinalidade na APS do município de Rio Verde, que são estratégicas para gestores e profissionais, a APS está em ampla expansão na cidade e no Brasil e avaliar sua qualidade é fundamental para garantir a legitimidade desta ampliação.

\section{AGRADECIMENTOS E FINANCIAMENTO}

Agradecemos a Universidade de Rio Verde e a Secretaria Municipal de Saúde de Rio Verde pelo apoio na realização da pesquisa.

\section{REFERÊNCIAS}

1. ALMEIDA MCP, MISHIMA SM. O desafio do trabalho em equipe na atenção à Saúde da Família: construindo "novas autonomias" no trabalho. Interface (Botucatu), Botucatu, 2001; 5(9): 150-153.

2. BARATIERI T, MARCON SS. Longitudinalidade no trabalho do enfermeiro: Identificando dificuldades e perspectivas de transformações. Texto Contexto Enfermagem, Florianópolis, 2012; 21(3): 549-57.

3. BRASIL. 2013. In: Ministério da Saúde. Conselho Nacional de Saúde. Resolução no 466 de 12 de dezembro de 2012. Diretrizes e normas regulamentadoras de pesquisas envolvendo seres humanos. Diário Oficial da União. Brasília.

4. BRASILIA. 2010, In: Ministério da saúde, Manual do Instrumento de Avaliação da Atenção Primária à Saúde, Brasília DF.

5. CUNHA EM, GIOVANELLA L. Longitudinalidade/continuidade do cuidado: identificando dimensões e variáveis para a avaliação da Atenção Primária no contexto do sistema público de saúde brasileiro. Ciência \& Saúde Coletiva, Rio de Janeiro, 2011; 16(1):1029-1042.

6. DIARIO OFICIAL. 2006 in: PORTARIA № 2.488, DE 21 DE OUTUBRO DE 2011. disponível em: <http://bvsms.saude.gov.br/bvs/saudelegis/gm/2006/prt0648_28_03_2006.html> acessado em 11 de junho de 2020

7. ELIAS PE, et al. Atenção Básica em Saúde: comparação entre PSF e UBS por estrato de exclusão social no município de São Paulo. Ciênc. Saúde Coletiva. 2006;11(3): 633-41

8. FIGUEIREDO AM. Avaliação da atenção primária à saúde: análise de concordância entre os instrumentos AMQ e PCATool no município de Curitiba, Paraná. Porto Alegre; 2011. dissertação (Mestrado profissional em epidemiologia-gestão de tecnologias em saúde) - Universidade Federal do Rio Grande do Sul, Porto alegre, 2011; 31-32p

9. GOIAS. 2018. In: Secretaria de estado de Gestão e planejamento. Caderno de Resultados. Goiânia. Disponível em: http://www.sgc.goias.gov.br/upload/arquivos/2018-02/03---atencao-basica-vsweb.pdf. acessado em 11 jan. 2020.

10. GOMES MFP, FRACOLLI LA. Avaliação da estratégia saúde da família sob a ótica dos profissionais. Revista Brasileira em Promoção da Saúde, 2018; 31(3): 1-13

11. HAUSER L, et al. Tradução, adaptação, validade e medidas de fidedignidade do instrumento de avaliação da atenção primária à saúde (PCATool) no Brasil: versão profissionais de saúde. Rev Bras Med Farm Comunidade. 2013; 8(29): 244-55.

12. LEÃO CDA, CALDEIRA AP. Avaliação da associação entre qualificação de médicos e enfermeiros em atenção primária em saúde e qualidade da atenção. Ciência \& Saúde Coletiva, 2011; 16(11): 4415-4423.

13. LINARD AG, et al. Integralidade da assistência na compreensão dos profissionais da Estratégia Saúde da Família. Rev. Gaúcha Enferm. 2011; 32(3): 546-53.

14. MINISTERIO DA SAUDE. 2002. In: Seminário Internacional Tendências e Desafios dos Sistemas de Saúde nas Américas São Paulo, O sistema público de saúde Brasileiro. Disponível em http://bvsms.saude.gov.br/bvs/publicacoes/sistema_saude.pdf. acesso em 10 jan. 2020.

15. OLIVEIRA MPR, et al. Training and Qualification of Health Professionals: Factors Associated to the Quality of Primary Care. Rev. bras. educ. med., Rio de Janeiro, 2016; 40(4): 547-559.

16. PRATES ML, et al. Performance of primary health care according to PCATool instrument: a systematic review. Ciênc. saúde coletiva, Rio de Janeiro, 2017; 22(6): 1881-1893.

17. RIBEIRO LCC, et al. Acolhimento às crianças na atenção primária à saúde: um estudo sobre a postura dos profissionais das equipes de saúde da família. Cad. Saúde Pública, Rio de Janeiro, 2010; 26(12): 2316-2322.

18. STARFIELD B, Atenção primária: equilíbrio entre necessidades de saúde, serviços e tecnologia. In: Atenção Primária: equilíbrio entre necessidades de saúde, serviços e tecnologia. 2004; $272 p$

19. SILVA FM, et al. Contribuições de grupos de educação em saúde para o saber de pessoas com hipertensão. Rev. bras. enferm., Brasília, 2014; 67(3): 347-353.

20. TURCI MA, et al. The influence of structural and organizational factors on the performance of primary health care in Belo Horizonte, Minas Gerais State, Brazil, according to nurses and managers.Cad. Saúde Pública, Rio de Janeiro, 2015; 31(9): 1941-1952. 